\title{
Behavioral dynamics of the affordance "graspable"
}

\author{
Stacy M. Lopresti-Goodman • Michael T. Turvey • \\ Till D. Frank
}

Published online: 21 June 2011

(C) Psychonomic Society, Inc. 2011

\begin{abstract}
On a daily basis, one perceives whether an object affords grasping with one hand or with both hands. In experiments in which differently sized objects of a fixed type have been presented, the transition from using one manual mode to the other has depended on both the ratio of object size to hand span and the presentation sequence - that is, size increasing versus decreasing. The transitions and their observed hysteresis (i.e., a transition ratio larger for the increasing sequence) can be accommodated by the order parameter dynamics typifying self-organizing systems. Here, we show that hysteresis magnitude depends on (a) the interaction between the attractors (one hand vs. two hands) and (b) the strength of the two-hands attractor. Through modeling and experimental results, we extend the investigation of affordance perception within dynamical-systems theory.
\end{abstract}

Keywords Affordances · Grasping · Dynamic modeling · Hysteresis · Cognitive task

In our everyday interactions with the environment, we are confronted with objects that may be grasped with either one or two hands. For example, one might perceive that the nearby empty coffee mug can be grasped with one hand, but that the nearby empty salad bowl must be grasped with two hands, given its greater width. To perceive an

S. M. Lopresti-Goodman $(\bowtie)$

Department of Psychology, Marymount University, 2807 North Glebe Road, Arlington, VA 22207, USA

e-mail: Stacy.Lopresti-Goodman@marymount.edu

M. T. Turvey • T. D. Frank

Center for the Ecological Study of Perception and Action,

University of Connecticut,

Storrs, CT, USA opportunity for action (e.g., to grasp and hold) is, in Gibson's (1979) terms, to perceive an affordance: to perceive what the current layout of surfaces affords in respect to one's dimensions and action capabilities (e.g., hand size, strength). For example, the coffee mug that affords grasping with one hand by an adult only affords grasping with two hands by a small child. Despite the different action capabilities of differently sized individuals, a dimensionless object-size/hand-span ratio, known as a pi number (Warren, 1984), invariantly specifies the boundary between one-hand and two-hand grasping for light objects. This boundary differs experimentally, however, for systematically increasing and systematically decreasing the pi number: That is, there is hysteresis (Lopresti-Goodman, Richardson, Baron, Carello, \& Marsh, 2009; Richardson, Marsh, \& Baron, 2007; van der Kamp, Savelsbergh, \& Davis, 1998). The goal of the present study is to advance experimental investigations of affordances by incorporating such investigations within a dynamical systems framework (e.g., Warren, 2006). This study centers on a dynamical model of behavioral transitions in grasping (Frank, Richardson, LoprestiGoodman, \& Turvey, 2009), hereafter referred to as the grasping-transition (GT) model.

\section{A pattern formation/recognition framework}

The GT model is derivative of Haken's (1991) multistable competitive model for pattern formation and recognition. Frequently, self-organizing dynamic systems are modeled as spatiotemporal evolution equations. These can be decomposed into equations that describe the evolution of the system's modes, which can be spatiotemporal objects themselves, and the evolution of the system's 
amplitudes, which tell us whether a particular mode out of the set of possible modes is present or absent (see Fig. 1). Haken $(1988,1991)$ argued that the amplitude equations alone form a closed description of the system. In the Bénard instability in fluid dynamics, for example, where a thin layer of fluid is gradually heated from below, convection roll patterns spontaneously emerge in the fluid due to temperature differences. The modes of this system are the roll patterns of certain orientations and wave length (i.e., measure for characterizing the "width" of a convection roll). Roll patterns that have the same "width" but differ in orientation compete with one another; however, only one of the competing patterns emerges. In this sense, the fluid layer acts as a "winner-takes-all" system. For the Bénard instability, the amplitude equations, including all parameters (coefficients), have been determined explicitly from the underlying spatiotemporal evolution equations (Bestehorn \& Friedrich, 1999; Bestehorn, Friedrich, \& Haken, 1989; Bestehorn \& Haken, 1991). The amplitude equations of this multistable competitive system are those of Haken's pattern recognition model (Bestehorn \& Haken, 1991). This kind of approach, which starts with the spatiotemporal evolution equations, is called a bottom-up approach.

In the field of cognition, perception, action, and memory, it is often difficult to carry out such a bottom-up approach. It is plausible to assume, however, that the systems under consideration belong to the class of multistable selforganizing systems. Based upon this assumption, top-down approaches that exploit Haken's amplitude equations have been carried out (Bressloff \& Roper, 1998; Ditzinger, Tuller, Haken, \& Kelso, 1997; Frank, 2009; Fuchs \& Haken, 1988a, 1988b; Haken, 1988). In particular, when dealing with only two competing modes, the multistable model reduces to a bistable model. The bistable competitive model for pattern recognition has been used for dynamical analyses of the perceptual oscillations induced by viewing a bistable figure, such as the ambiguous face-vase image (cf. Rubin, 1915; for other examples of dynamical models of the perception of ambiguous figures, see also Ditzinger \& Haken, 1989; Merk \& Schnakenberg, 2002; Nagao, Haruhiko, \& Matsui, 2000), given that only one stable perception may exist at a time (e.g., either the face or the vase is perceived).

\section{The GT model and affordances in preview}

Although the GT model was developed to address transitions in a bistable behavioral domain-namely, grasping with either one or two hands - it is not limited to that domain. It can be appropriated to address key aspects of behavioral transitions in affordance experiments more generally (cf. Fitzpatrick, Carello, Schmidt, \& Corey, 1994; Hirose \& Nishio, 2001), such as hysteresis and the distinction between (a) transitions induced biomechanically and (b) transitions induced by interacting behavioral modes. A significant motivation for modeling the particular affordance "graspable" is the frequency with which hysteretic grasping transitions are observed (Cesari \& Newell, 1999, 2000; Lopresti-Goodman et al., 2009; Newell, Scully, Tenenbaum, \& Hardiman, 1989; Richardson et al., 2007; van der Kamp et al., 1998).

Experiments covering a variety of affordances have demonstrated that the shift from one mode of behavior to another exhibits features typical of a self-organized dynamic system (Fitzpatrick et al., 1994; Hirose \& Nishio, 2001; Lopresti-Goodman et al., 2009; Richardson et al., 2007; van der Kamp et al., 1998), where stable macroscopic patterns of behavior emerge from the lawful interactions among system components (Kelso, 1995). ${ }^{1}$ The different types of behaviors exhibited (e.g., one- or two-handed grasps) represent the system's stable or ordered states and may be considered collective variables or order parameters $(\xi)$ of the dynamic system. In the present study, the dimensionless object-size/hand-span ratio, the grasping pi number, acts as a control parameter $(\alpha)$ for the two variants (one hand, two hands) of the dynamic system's order parameter (Fitzpatrick et al., 1994; van der Kamp et al., 1998; Warren, 1984). In general terms, an order parameter undergoes a spontaneous transition when its control parameter passes through a critical value, $\alpha_{\mathrm{c}}$ (cf. Haken, 1988; Strogatz, 1994). A familiar example is interlimb rhythmic coordination, with relative phase as the order parameter and movement frequency as the control parameter (Kelso, 1995). With increasing frequency, an initially antiphase interlimb organization (relative phase of $180^{\circ}$ ) shifts abruptly to the more stable in-phase interlimb organization (relative phase of $0^{\circ}$ ).

Hysteresis is a hallmark characteristic of nonlinear self-organizing systems-a sensitivity to initial conditions (in the preceding example, starting at $0^{\circ}$ or $180^{\circ}$ ) that manifests as a dependence of $\alpha_{\mathrm{c}}$ on the direction of scaling $\alpha$. The magnitude of $\alpha_{\mathrm{c}}$ is typically less when the series of $\alpha$ values progresses from the largest to the smallest value (descending sequence) than when change is in the opposite direction (ascending sequence). In grasping experiments, where the perception of grasping is indexed

\footnotetext{
${ }^{1}$ With regard to the self-organized dynamics of animal behavior, the components comprising the animal do not suffice. The components must be those comprising the animal-environment-task system (Beer, 1995a, 1995b, 2009; Chemero \& Turvey, 2007; Järvilheto, 1998, 2009; Richardson, Shockley, Fajen, Riley, \& Turvey, 2008; Warren, 2006).
} 


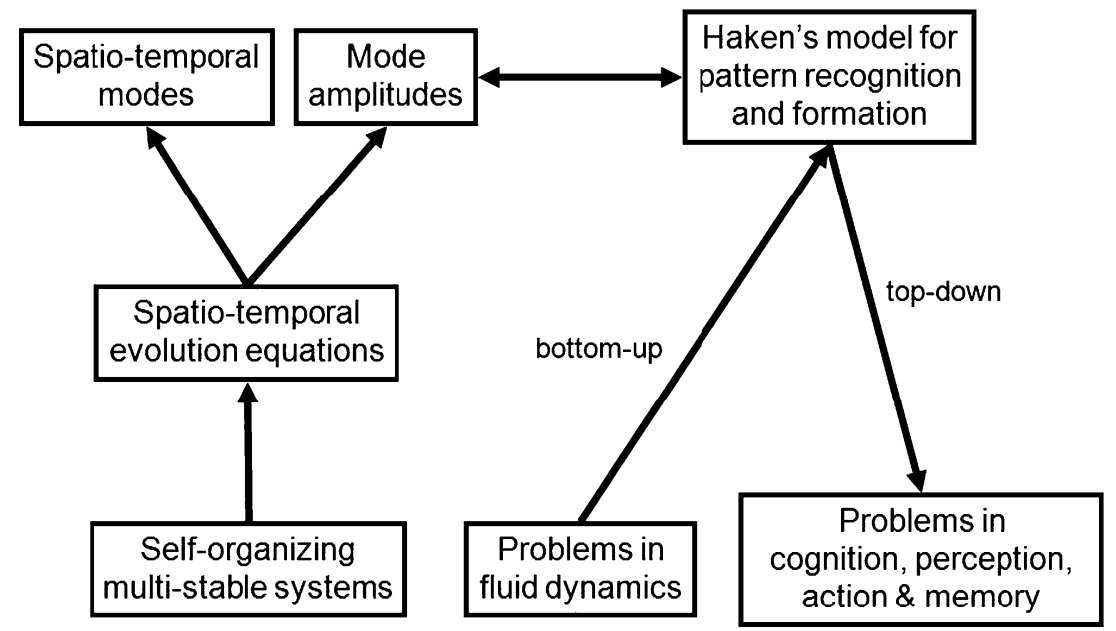

Fig. 1 Schematic illustration of the generality of Haken's pattern formation and recognition model. Left: Self-organizing multistable systems frequently can be best understood by representing the spatiotemporal dynamics in terms of modes and mode amplitudes. The mode amplitudes quantify the extents to which modes have emerged. Link between left and right: Haken's model describes the evolution of mode amplitudes for a general class of multistable systems. Right: The model equations can explicitly be derived in a bottom-up approach from the microscopic equations determining problems in fluid dynamics. Conversely, Haken's model has turned out to be a fruitful top-down approach for understanding problems in cognition, perception, action and memory by selective action - that is, actually performing one- and two-hand grasps-positive hysteresis ( $\alpha_{\mathrm{c}}$ for ascending sequences is greater than $\alpha_{\mathrm{c}}$ for the descending sequences) is the common finding (Lopresti-Goodman et al., 2009; Richardson et al., 2007; van der Kamp et al., 1998). In contrast, negative hysteresis (or enhanced contrast; Tuller, Case, Ding, \& Kelso, 1994) is typically seen in experimental contexts when perception is indexed by selective verbal classification (e.g., "two hands," "not step-overable," "stand-on-able"; Fitzpatrick et al., 1994; Hirose \& Nishio, 2001; Pufall \& Dunbar, 1992; Richardson et al., 2007). A third possible scenario is critical point: The transition occurs at the same $\alpha_{\mathrm{c}}$, regardless of the direction of scaling of $\alpha$ (Tuller et al., 1994).

In grasping experiments, the one-to-two-hand transition in the ascending sequences is biomechanically induced: When the object-size/hand-span ratio $(\alpha)$ exceeds unity, object dimensions become larger than body dimensions and a transition from one- to two-hand grasping must occur, because the object can no longer be grasped with one hand..$^{2}$ In contrast, the two-to-one-hand

\footnotetext{
$\overline{{ }^{2} \text { In principle, }}$ it is assumed that participants perceive the physical constraint at $\alpha<1$. This is reflected in empirical studies on grasping that have demonstrated $\alpha<1$ for the ascending series (cf. LoprestiGoodman et al., 2009; Richardson et al., 2007). It is also possible, however, that $\alpha=1$ is a sufficient but not necessary condition for the vanishing of the one-hand mode in the ascending sequences. That is, there may be a transition from one to two hands at $\alpha<1$ due to the competition between the one- and two-hand modes.
}

transition in the descending sequences is hypothesized to occur due to an interaction of, and competition between, the two possible behavioral modes. This is because in the descending sequences, no biomechanical constraint exists that forces participants to switch from two hands to one, since the two-hand mode is a possible behavioral mode for all object sizes. Experiments on grasping have shown, however, that the majority of participants in the descending sequences do switch to the one-hand mode for smaller objects (Cesari \& Newell, 1999, 2000; LoprestiGoodman et al., 2009; Newell et al., 1989; Richardson et al., 2007; van der Kamp et al., 1998). Therefore, it must be the interaction of the two possible modes and the necessity of mode selection given the formulation of the "winner takes all" dynamics that triggers the two-to-one-hand transitions in the descending sequences. It is important that the GT model be able to distinguish between transitions that are brought about as a result of biomechanical limitations and those that are brought about by competing behavioral modes.

\section{The GT model and its predictions}

Let the two variants of the order parameter, $\xi_{1}$ and $\xi_{2}$, represent the generalized amplitudes of the one- and twohand grasping modes, respectively. In line with the Frank et al. (2009) model, we will only consider positive values of $\xi$; therefore, $\xi_{1}>0, \xi_{2}=0$ defines the one-hand mode, and $\xi_{2}>0, \xi_{1}=0$ defines the two-hand mode. Then, the 
grasping behavior is determined by the time evolution of $\xi_{1}$ and $\xi_{2}$ :

$\dot{\xi}_{1}(t)=\lambda_{1} \xi_{1}-B \xi_{2}^{2} \xi_{1}-C\left(\xi_{1}^{2}+\xi_{2}^{2}\right) \xi_{1}$,

and

$\dot{\xi}_{2}(t)=\lambda_{2} \xi_{2}-B \xi_{1}^{2} \xi_{2}-C\left(\xi_{1}^{2}+\xi_{2}^{2}\right) \xi_{2}$.

In Eqs. (1) and (2), the terms $B>0$ and $C>0$ represent the magnitudes of the system's nonlinear interactions. The larger the values of $B$ and $C$, the greater the interaction between the two grasping modes, $\xi_{1}$ and $\xi_{2}$. The nature of the interaction is two-fold: It results in both competition and saturation. Below, we will transform Eqs. (1) and (2) such that these two different kinds of effects can be identified more explicitly. The parameters $\lambda_{1}$ and $\lambda_{2}$ are the one- and two-hand variants of what can be termed "availability" parameters corresponding, respectively, to $\xi_{1}$ and $\xi_{2}$. In the Haken recognition model, a pattern can only be available (and therefore attended to or recognized, in the conventional terminology of the model) if $\lambda>0$. The $\lambda$ parameter determines the relaxation time to the stationary fixed point, $\xi_{\text {st }}$, after a perturbation. The larger the value of $\lambda$, the faster the relaxation time to $\xi_{\mathrm{st}}$, and therefore the more stable that behavior is. In line with the original conception, the grasping mode may be available for $\lambda>0$, and may not be available for $\lambda<0$.

Equations (1) and (2) can be expressed in terms of their potential dynamics

$\frac{d}{d t} \xi_{1}(t)=-\frac{\partial}{\partial \xi_{1}} V\left(\xi_{1}, \xi_{2}\right)$

and

$\frac{d}{d t} \xi_{2}(t)=-\frac{\partial}{\partial \xi_{2}} V\left(\xi_{1}, \xi_{2}\right)$

involving the two-dimensional potential

$V\left(\xi_{1}, \xi_{2}\right)=-\frac{1}{2}\left(\lambda_{1} \xi_{1}^{2}+\lambda_{2} \xi_{2}^{2}\right)+\frac{B}{2} \xi_{1}^{2} \xi_{2}^{2}+\frac{C}{4}\left(\xi_{1}^{2}+\xi_{2}^{2}\right)^{2}$.

Note that in our exposition, we will not evaluate the magnitudes of $\xi_{1}$ and $\xi_{2}$, but instead will focus only on whether a behavioral mode is present (e.g., $\xi_{1}>0$ ) or absent (e.g., $\xi_{1}=0$ ).

The potentials of the GT model are related to the availability parameters. As discussed above, the larger and more positive the value of $\lambda$, the more stable the attractor representing a behavioral mode and the faster the relaxation time to $\xi_{\text {st }}$ after a perturbation. This is illustrated in Fig. 2 by steeper potential wells or minima. Conversely, the smaller the value of $\lambda$, the less stable the attractor representing the behavioral mode. This is illustrated in
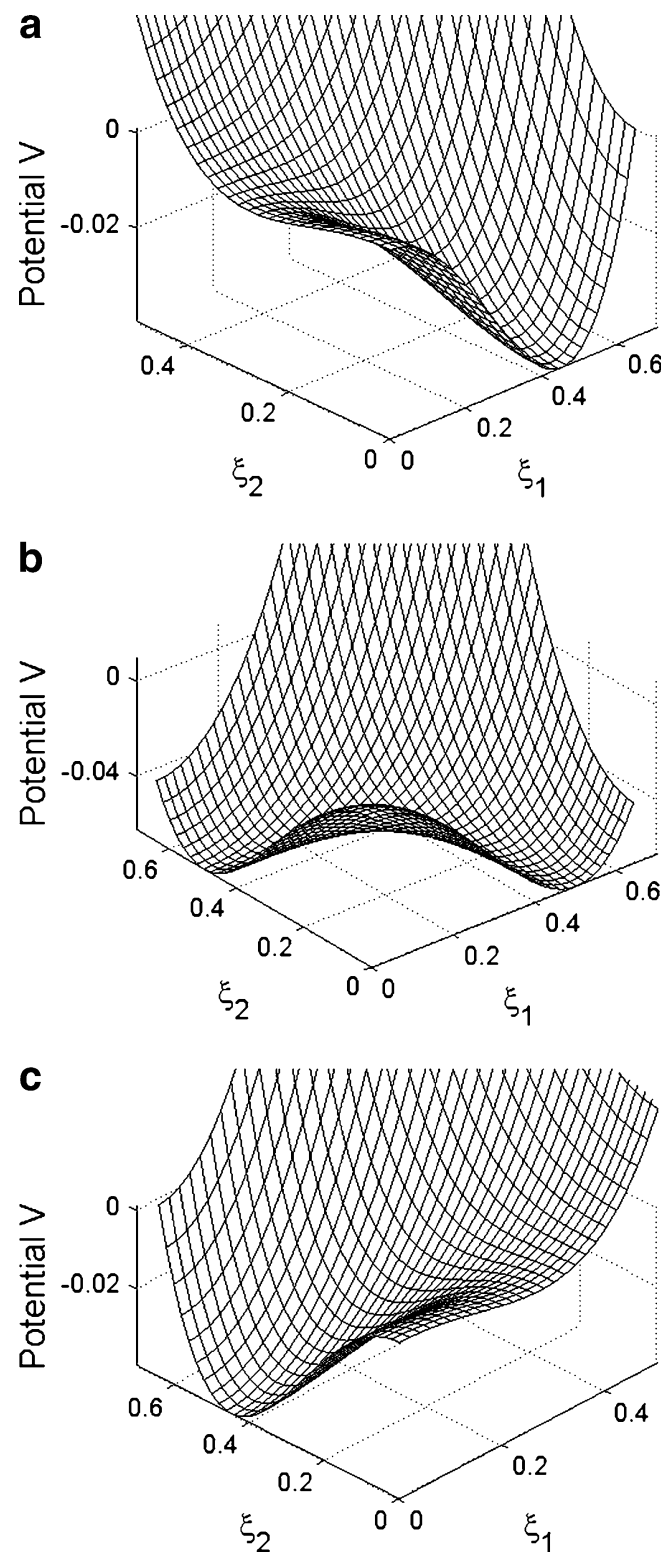

Fig. 2 The two-dimensional potentials for Eq. 5. (a) The monostable case where one-hand grasps would be performed; (b) the bistable case, where the grasping behavior performed depends on the direction of scaling $\alpha$; and (c) the monostable case where two-hand grasps would be performed. Potential minima represent stable states, while potential maxima represent unstable states

Fig. 2 by potential maxima. Figure 2 illustrates the potentials for three fixed points derived from Eqs. (1) and (2). Figure 2a illustrates the case where $\lambda_{1}>>\lambda_{2}$. In this situation, linear stability analysis shows that for any initial condition $\xi_{1}(0)>0, \xi_{2}(0)>0$, the system will converge to the stable mode $\xi_{1}$ and away from the unstable mode $\xi_{2}$, which is a repellor. In this situation, one-hand grasps will be performed. Figure $2 \mathrm{~b}$ illustrates the case where $\lambda_{1} \approx \lambda_{2}$. Linear stability analysis shows that in this situation the system is bistable. Given the winner-takes-all formulation 
of the model, one- and two-hand grasps cannot be performed simultaneously, and therefore the behavior exhibited depends on the direction of scaling $\alpha$. Figure 2c illustrates the situation where $\lambda_{2} \gg \lambda_{1}$. Linear stability analysis shows that for any initial condition $\xi_{1}(0)>0$, $\xi_{2}(0)>0$, the system will converge to the stable mode $\xi_{2}$ and away from the unstable mode $\xi_{1}$. In this situation, two-handed grasps will be performed.

With regard to the two aforementioned types of behavioral transitions, in the ascending sequences a switch due to biomechanical constraints from one to two hands is made when $\lambda_{1} \leq 0$. In general (i.e., when considering both ascending and descending sequences), a switch from one mode to another occurs when $\lambda_{2}>(1+B / C) \lambda_{1}$ (transition from one- to two-hand grasps) or when $\lambda_{1}>(1+B / C) \lambda_{2}$ (transition from two- to one-hand grasps). According to Eq. (12) of Frank et al. (2009), we assume that a linear relationship exists between $\lambda_{1}$ and $\lambda_{2}$ and the control parameter $\alpha$ (see Fig. 3):

$\lambda_{1}=\beta(1-\alpha)$,

$\lambda_{2}=\lambda_{2,0}+\beta \alpha$,

where $\beta$ determines the overall size of the availability parameters and $\lambda_{2,0}$ represents the initial value of the twohand availability parameter. The thin decreasing line in Fig. 3 illustrates how $\lambda_{1}$ decreases in strength as $\alpha$ increases in value, a relationship determined by the minus sign in Eq. (6). When $\lambda_{1}=0, \alpha$ assumes a value of 1 , and at this point one-hand grasping is no longer possible due to biomechanical constraints; therefore, a switch to two-hand grasping must be made. Conversely, the dark increasing line in Fig. 3

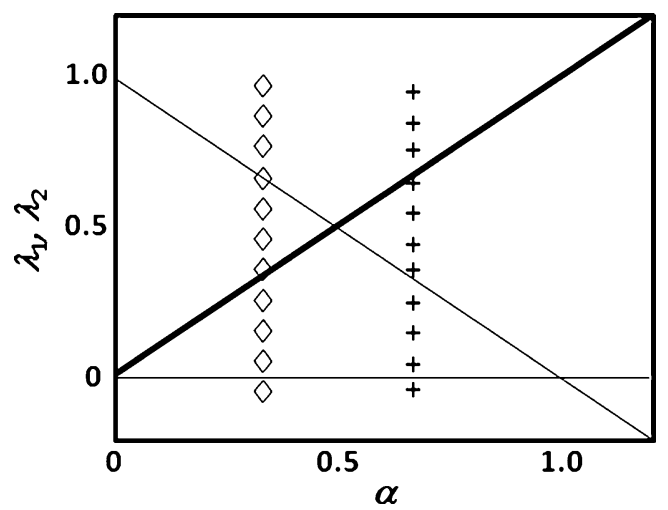

Fig. 3 Qualitative plot representing the relationship between the control parameter $\alpha$ and the one- and two-hand availability parameters $\lambda_{1}$ (thin line decreasing) and $\lambda_{2}$ (thick line increasing), respectively, for $B=C=1, \lambda_{2,0}=0, \beta=1$ (which implies that $g=1+B / C=2$ ). In the ascending sequences, the transition from one to two hands occurs at the critical value of $\alpha, \alpha_{\mathrm{c}, 2}$ (crosses), when $\lambda_{2}>(1+B / C) \lambda_{1}$. In the descending sequences, the transition from two hands to one occurs at $\alpha_{\mathrm{c}, 1}$ (diamonds) when $\lambda_{1}>(1+B / C) \lambda_{2}$ illustrates how $\lambda_{2}$ increases in strength with increases in $\alpha$, a relationship determined by the plus sign in Eq. (7). Note that Eq. (6) guarantees that for $\alpha$ values larger than unity, we have $\lambda_{1}<0$ (as required above).

The GT model, as presented in Eqs. (1) and (2), is defined in terms of two first-order dynamic equations involving two collective variables, $\xi_{1}$ and $\xi_{2}$, and four parameters, $B, C, \lambda_{2,0}$, and $\beta$. In grasping-transition experiments, typically two dependent variables are observed: the $\alpha_{\mathrm{c}}$ values in the ascending $\left(\alpha_{\mathrm{c}, 2}\right)$ and descending $\left(\alpha_{\mathrm{c}, 1}\right)$ trials. Our objective is to determine model parameters from these two observations. Consequently, only two parameters can be estimated. In order to see which of the parameters can be determined, we exploit the fact that in many cases, such models can be simplified (i.e., parameters can be eliminated) by rescaling time and by introducing appropriately defined rescaled variables (cf. Murray, 1993). In this context, we first note that given that $\beta$ strictly determines the overall size of the availability parameters, it seems that we can simplify Eqs. (6) and (7) by defining the availability parameters scaled relative to $\beta$ as

$\lambda_{1} / \beta=1-\alpha$,

$\lambda_{2} / \beta=L_{2,0}+\alpha$,

with the initial value of the rescaled availability parameter, $L_{2,0}$, calculated as $\lambda_{2,0} / \beta$.

In our case, we rescale time with the parameter $\beta$ and introduce another parameter, $g$, that relates the two nonlinear interaction terms $B$ and $C$ from Eqs. (1) and (2). ${ }^{3}$

$g=1+B / C$, or $B=C(g-1)$.

Subsequently, we rescale the generalized amplitudes $\xi_{1}$ and $\xi_{2}$ with the square root of $C / \beta$. A detailed calculation (see Appx. A) shows that Eqs. (1) and (2) become

$\dot{\xi}_{1}=\lambda_{1} \xi_{1}-g \xi_{2}^{2} \xi_{1}-\xi_{1}^{3}$,

and

$\dot{\xi}_{2}=\lambda_{2} \xi_{2}-g \xi_{1}^{2} \xi_{2}-\xi_{2}^{3}$,

with $\lambda_{1}=1-\alpha$ and $\lambda_{2}=L_{2,0}+\alpha$. In short, we have reduced the four-parameter model to a model that involves only two parameters, namely $g$ and $L_{2,0}$ (in addition to the control parameter $\alpha$ ). From Eqs. (11) and (12), we see that

\footnotetext{
${ }^{3}$ Calculation of the parameter $C$ depends on knowing the values of the stationary order parameters, $\xi_{\text {st }}$. Since their true values are unknowable, it is not possible to obtain a value for $C$. The parameter $\beta$ depends on how the order parameter relaxes to its stationary value $\xi_{\text {st }}$. This would require measurement of the temporal evolution of the order parameter, which is beyond the scope of the present study.
} 
$g$ is the coefficient that occurs in the mixed terms. Accordingly, the parameter $g$ represents the strength of the competitive interaction (not related to saturation effects) between the one- and two-hand grasping modes. Saturation effects are described by the cubic $\xi_{1}$ and $\xi_{2}$ terms.

Let us briefly address some interpretations and predictions of the GT model relevant to the present study. First, it can be shown that the stability of the grasping modes, as measured by the Lyapunov exponents obtained from a linear stability analysis, increase linearly with $g$ (Frank et al., 2009). Consequently, the larger the values of $g$, the more stable the particular solution to the equation. Therefore, we may consider $g$ as a measure of the strength of the system's attractor. Second, if we plot $\lambda_{1}=1-\alpha$ and $\lambda_{2}=L_{2,0}+\alpha$ on a logarithmic scale as a function of parameter $\alpha$, we can graphically determine the hysteresis size, as shown in Fig. 4a. To this end, we create a shifted max- $\lambda$ line: We plot separate lines at distances of $\log (g)$ below the $\lambda$ value that is the larger of $\lambda_{1}$ and $\lambda_{2}$. In Fig. 4a, this shifted max- $\lambda$ line is shown for two different values of $g$. The hysteresis size is then given by the intersection points between the graphs for $\lambda_{1}$ and $\lambda_{2}$ and the shifted max- $\lambda$ line (for further details, see Frank, 2009; Frank, van der Kamp, \& Savelsbergh, 2010). By this construction, it becomes obvious that when $g$ increases, the hysteresis size increases. In sum, the GT model predicts that interaction strength, attractor stability, and hysteresis size are closely related properties and scale in the same direction. In particular, $g$ is related to the degree of hysteresis.

In Fig. $4 b, \lambda_{2}$ is plotted on a linear scale as a function of $\alpha$ for two different values of $L_{2,0}$. If $L_{2,0}$ is positive and large, the overall "availability" of the two-handed grasping mode is large as well. In contrast, if $L_{2,0}$ assumes a small positive value or becomes negative, the overall "availability" of grasping with two hands is relatively low. Therefore, the GT model predicts that the availability of the two-handed grasping mode will "affect," or will be reflected by variations in, the parameter $L_{2,0}$. The parameters $L_{2,0}$ and $g$ can be derived from the mean pi numbers $\left(\alpha_{\mathrm{c}}\right)$ in the ascending and descending sequences, as shown in Appendix B.

In overview, the present article is an evaluation of the GT model. The evaluation is conducted in two phases. In Phase 1, we derive parameter estimates for the model from the data of previously conducted experiments. These experiments by Lopresti-Goodman et al. (2009) examined how grasping transitions (indexed by selective action) were affected by the difficulty of the task. In Phase 2, we present a new experiment to test predictions of the effects of task difficulty derived from Phase 1. The new experiment provides data on transitions in selective action in the context of a concurrent cognitive task with four levels of difficulty.
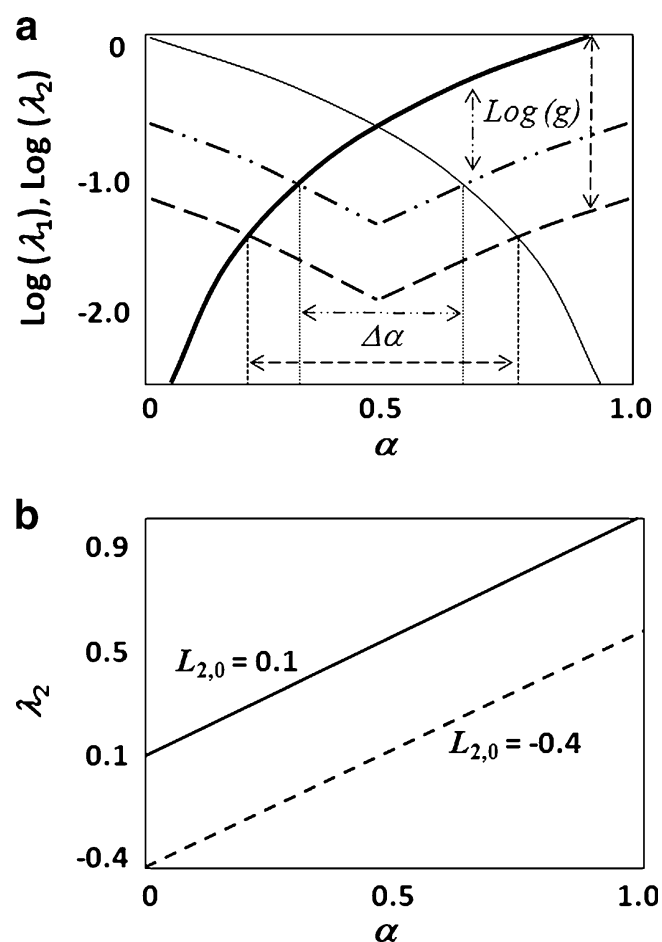

Fig. 4 (a) Availability parameters $\lambda_{1}$ and $\lambda_{2}$ as functions of the control parameter $\alpha$ as in Fig. 3, but here depicted in a log-linear plot for two different values of $g$. The thin decreasing line represents $\log$ $\left(\lambda_{1}\right)$ versus $\alpha$, while the thick increasing line represents $\log \left(\lambda_{2}\right)$ versus $\alpha$. The horizontal arrows show the hysteresis size $(\Delta \alpha)$ for two different values of $g$, while the vertical arrows represent the interaction strength measured on a logarithmic scale [i.e., in terms of $\log (g)]$ for the two values of $g$. By construction, we see that when $g$ increases (vertical arrows become longer), $\Delta \alpha$ increases (horizontal arrows become longer), as well. (b) Availability of two-handed grasping as a function of the control parameter $\alpha$ for two different values of the offset parameter $L_{2,0}$. The diagram illustrates that the availability of two-hand grasping is greater for the larger of the two offset parameters, irrespective of $\alpha$

\section{Phase 1: Model-based analysis of Lopresti-Goodman} et al. (2009)

Experiments 1 and 2 of Lopresti-Goodman et al. (2009) investigated the task of grasping and moving light wooden planks of different sizes, using either one or two hands in ascending and descending presentation sequences. The wooden planks were $2 \mathrm{~cm}$ high and $6.5 \mathrm{~cm}$ wide and ranged in length from 3 to $30 \mathrm{~cm}$ in $1-\mathrm{cm}$ increments in Experiment 1, and from 4.5 to $24.5 \mathrm{~cm}$ in $0.5-\mathrm{cm}$ increments in Experiment 2. All planks were painted black with their ends red. Participants were instructed that they could only grasp a plank by its red ends, which restricted them to grasping the planks lengthwise.

Experiment 1 of Lopresti-Goodman et al. (2009) assessed the influences of three different presentation speeds on the point of transition between one- and two- 
hand grasping $(n=23){ }^{4}$ The fast ( $3 \mathrm{~s}$ per plank) and selfpaced ( $\sim 5 \mathrm{~s}$ per plank) presentation speeds yielded positive hysteresis, with the critical values of the control parameter in the descending sequence, $\alpha_{\mathrm{c}, 1}$, being much smaller than those for the ascending sequence, $\alpha_{\mathrm{c}, 2}\left(\alpha_{\mathrm{c}, 1}<\alpha_{\mathrm{c}, 2}\right)$. In the slowest presentation condition (10 s per plank), positive hysteresis was eliminated. Instead, a critical-point transition occurred $\left(\alpha_{\mathrm{c}, 1}=\alpha_{\mathrm{c}, 2}\right)$.

Experiment 2 of Lopresti-Goodman et al. $(2009 ; n=48)$ investigated the influence of a cognitive load, a simple arithmetic task of counting back from a random three-digit number by 7 , for fast-paced and slow-paced presentation speeds. The addition of the cognitive load exaggerated positive hysteresis in both speed conditions $\left(\alpha_{\mathrm{c}, 1}<<\alpha_{\mathrm{c}, 2}\right)$, while more moderate positive hysteresis was seen in the noload conditions $\left(\alpha_{\mathfrak{c}, 1}<\alpha_{\mathfrak{c}, 2}\right)$.

As mentioned above, $g$ is related to the degree of hysteresis. A qualitative examination of the LoprestiGoodman et al. (2009) data revealed that positive hysteresis was exaggerated with increased task difficulty (e.g., a fast presentation speed or an additional cognitive load). Therefore, the GT model suggests that the $g$ parameter should increase with increased levels of task difficulty. Moreover, as demonstrated above, the $L_{2,0}$ parameter reflects the stability of the two-handed grasping mode. It is plausible to assume that when task difficulty increases, the two-handed grasping mode increases in stability since, biomechanically, it is a behavior that can always be performed even under the most difficult of cognitive-load conditions. If this is the case, this would imply that the value of $L_{2,0}$ increases with increases in task difficulty.

\section{Results of the reevaluation}

A univariate ANOVA on the $g$ parameter, as estimated from the Experiment 1 data, revealed that there was no significant effect of speed, $F(2,20)=2.34, p=.12, \eta^{2}=.19$, despite the $g$ parameter being larger for the self-paced $(M=1.43 \pm 0.70$, $\Delta \alpha=.06)$ and fast-paced $(M=1.60 \pm 0.65, \Delta \alpha=.12)$ presentation speed conditions than for the slow-paced condition $(M=0.98 \pm 0.28, \Delta \alpha=.00)$. A planned comparison $t$ test on the fast- and slow-paced presentation speed $g$ values did reveal, however, a significant difference, $t(13)=-2.43, p=.03$ (see Fig. 5a). This suggests that the interaction between the two possible grasping modes increased for the fast-paced condition, equivalently increasing the strength of both modes' attractors.

\footnotetext{
${ }^{4}$ A group of 24 participants from the University of Connecticut participated in Lopresti-Goodman et al.'s (2009) Experiment 1. One participant in the fast-paced condition, however, failed to grasp the objects properly, and for this reason the participant's data were excluded from the analysis.
}

A 2 (speed: slow or fast) $\times 2$ (cognitive load: no load or load) univariate ANOVA on the $g$ values of Experiment 2 revealed a main effect of speed, with $g$ for the fast-paced condition $(M=1.91 \pm 0.85, \Delta \alpha=.20)$ being significantly larger than $g$ for the slow-paced condition $(M=1.37 \pm 0.60$, $\Delta \alpha=.09), F(1,44)=6.28, p=.02, \eta^{2}=.13$ (see Fig. $5 b$ ). There were no significant differences for the different cognitive-load conditions, $F(1,44)=2.02, p=.16, \eta^{2}=.04$ $\left(M_{\text {Load }}=1.79 \pm 0.69, \Delta \alpha=.19\right.$, and $M_{\text {NoLoad }}=1.49 \pm 0.84$, $\Delta \alpha=.10$ ), nor was there a significant Speed $\times$ Cognitive Load interaction, $F<1$.

With regard to the $L_{2,0}$ parameter, a univariate ANOVA for Lopresti-Goodman et al.'s (2009) Experiment 1 data indicated no significant differences for the three speed conditions $\left(M_{\text {Slow }}=-0.44 \pm 0.26, M_{\text {Self }}=-0.48 \pm 0.16\right.$, and $\left.M_{\text {Fast }}=-0.38 \pm 0.23\right), F<1$. A univariate ANOVA of the Experiment 2 data revealed that there was a marginally significant main effect of cognitive load, $F(1,44)=2.79$, $p=.10, \eta^{2}=.06$, with the $L_{2,0}$ value being larger for the load $(M=-0.27 \pm 0.17, \Delta \alpha=.19)$ than for the no-load $(M=-0.36 \pm 0.21, \Delta \alpha=.10)$ condition (see Fig. 5c). There was not, however, a main effect of speed, nor an interaction between speed and cognitive load, both $F_{\mathrm{s}}<1$, despite differences seen in the $L_{2,0}$ values and the amounts of positive hysteresis exhibited for the different task difficulty conditions $\left(M_{\text {FastLoad }}=-0.22 \pm 0.16, \Delta \alpha=.24\right.$; $M_{\text {FastNoLoad }}=-0.35 \pm 0.20, \Delta \alpha=.15 ; M_{\text {SlowLoad }}=-0.31 \pm$ $0.16, \Delta \alpha=.13$; and $M_{\text {SlowNoLoad }}=-0.37 \pm 0.24, \Delta \alpha=.05$ ).

\section{Discussion of the reevaluation}

We may infer from the parameter derivations for $g$ from both Experiments 1 and 2 of Lopresti-Goodman et al. (2009) that increasing the speed of object presentation increases the interaction between the two grasping modes. The addition of the cognitive load-the simple arithmetic task - did not, however, significantly increase the interaction. With regard to the $L_{2,0}$ parameter, in both Experiments 1 and 2 the manipulation of speed did not influence its value. For Experiment 2, however, it appears that the $L_{2,0}$ parameter values tended to increase with increases in task difficulty. This suggests that the two-hand grasping mode may represent a more stable form of behavior (e.g., the attractor is stronger) when participants have to complete the cognitive task while grasping the experimental objects.

A closer inspection of the Lopresti-Goodman et al. (2009) data revealed that participants did not indiscriminately use the two-hand mode more often in the more difficult conditions (e.g., with increased speed or the addition of a cognitive load). Instead, the significant differences in $\alpha$ values reported by Lopresti-Goodman et al. for the Sequence $\times$ Speed interaction for Experiments 1 and 2, and the significant Sequence $\times$ Cognitive Load 

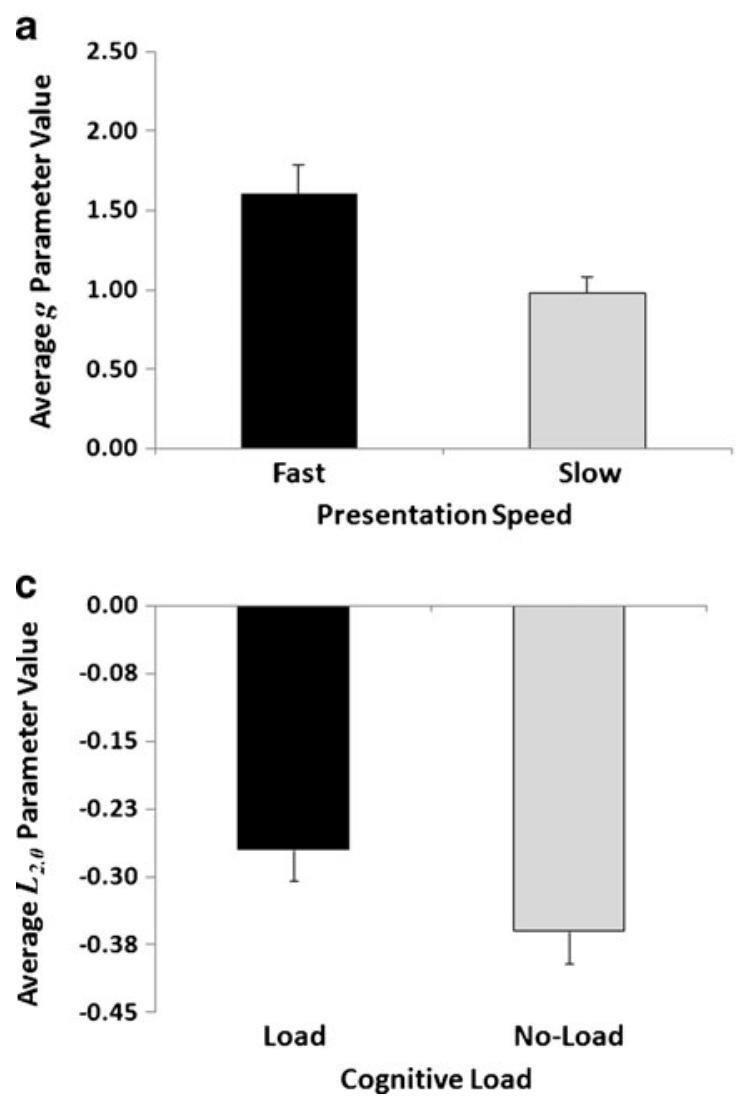

Fig. 5 The bars (with standard errors) represent the average $g$ parameter values derived for the slow-paced (gray) and fast-paced (black) presentation speed conditions from Experiments 1 (a) and 2 (b) of Lopresti-Goodman et al. (2009). In panel c, the bars (with

interaction for Experiment 2, indicate that participants only used the two-hand grasping mode more often in the descending sequences for more difficult conditions. Specifically, $\alpha_{c, 1}$ decreased with increases in task difficulty, whereas $\alpha_{\mathrm{c}, 2}$ was invariant, regardless of the level of task difficulty. This finding further supports the idea that participants tend to switch in the ascending sequences at their biomechanical limits $\left(\sim \alpha_{\mathrm{c}, 2}\right)$. Given these differences in the transition points for the different sequences, it is now clearer why significant changes in $L_{2,0}$ were not seen with changes in task difficulty.

In sum, the results of the parameter derivations from the Lopresti-Goodman et al. (2009) data indicate that the interaction of the two grasping modes increases with increasing task difficulty, specifically with increasing presentation speeds. This was revealed by the significant impact of the speed manipulation on the $g$ values for both Experiments 1 and 2. The addition of the cognitive load did not, however, increase the interaction between the grasping modes. It is possible that the manipulation of cognitive load in conjunction with the presentation speed manipulation

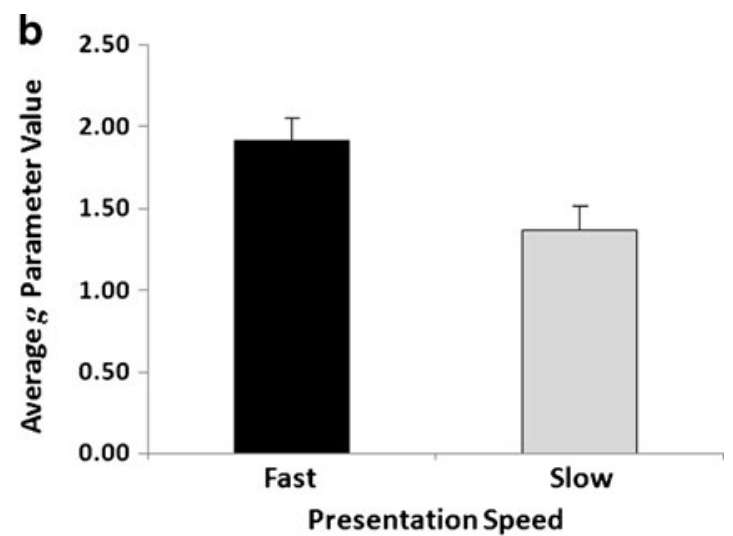

standard errors) represent the average $L_{2,0}$ parameter values derived for the cognitive load (black) and no cognitive-load (gray) conditions from Experiment 2 of Lopresti-Goodman et al

obscured any effect the cognitive load might have had when the participants performed the grasping task at a selfpaced speed. This is an evaluation to be tested in a new experiment presented below. Overall, the analyses suggest that the GT model parameters $g$ and $L_{2,0}$ are influenced by manipulations of task difficulty. Given this result, we can test predictions made by the GT model with regard to how other levels of task difficulty will influence the parameters.

\section{Phase 2: Evaluating GT model predictions about cognitive task difficulty}

The parameter derivations from the Lopresti-Goodman et al. (2009) data demonstrated that the GT model is capable of accounting for differences seen in the behavioral dynamics of grasping under parallel demands of increased speed of performance and/or cognitive load. By extension, the arithmetic activity of counting backward from an assigned number provides a means of systematically manipulating cognitive task difficulty. 
As argued and demonstrated by Posner (1964) and Posner and Rossman (1965), relatively uncomplicated arithmetic tasks allow for the equating of cognitive load with the amount of information reduced from a numerical stimulus (e.g., 12) to a response (e.g., " 3 " in reference to the sum of the two digits, and "odd" in reference to 3 ). In experiments directed at effects on rhythmic coordination of a parallel cognitive activity irrelevant to the coordination, Pellecchia and Turvey (2001) found evidence to suggest that the coordination's attractors shifted in a degree proportionate to the amount of information reduction quantifying the cognitive activity.

Of the several information reduction tasks evaluated by Posner and Rossman (1965), counting backward is most suited to the grasping-transition paradigm. This task can be performed continuously in parallel with the repetitive picking up and carrying of the planks (as shown in LoprestiGoodman et al., 2009, Exp. 2) and requires only that the starting number be given at a trial's outset. Four counting tasks were used in the GT model evaluation: (a) no counting (no concurrent cognitive task), (b) counting back by 1 (0-bit reduction), (c) counting back by 3 (5.93-bit reduction), or (d) counting back by 7 (7.62-bit reduction). Each variant of each of the counting tasks (b)-(d) started from a three-digit number between 900 and 999 (see Appx. C for details).

The expectation from the GT model was that participants in each of the counting conditions would exhibit positive hysteresis $\left(\alpha_{\mathrm{c}, 1}<\alpha_{\mathrm{c}, 2}\right)$, with positive hysteresis being largest for the most difficult condition (counting back by 7), smallest for the least difficult condition (no counting), and intermediate for the moderate-difficulty counting conditions (counting back by 1 , counting back by 3 ). The differences in the amounts of positive hysteresis exhibited should result from participants using the two-hand mode more often in the descending sequences for the more difficult tasks. It was also expected that participants in each condition would make the transition from one to two hands at similar $\alpha_{\mathrm{c}, 2}$ values in the ascending sequences, regardless of the counting condition they were in. Additionally, if increases in the difficulty of the counting task increase the amount of positive hysteresis, as suggested by the parameter derivations, then increases in the values of $L_{2,0}$ and $g$ would also be expected. Specifically, these parameters should be significantly larger for the more difficult than for the less difficult counting conditions.

Method

Participants A total of 37 individuals from the University of Connecticut volunteered to participate as partial fulfillment of a course requirement. Of those 37,32 individuals' data (16 males, 16 females; mean hand $\operatorname{span}=21.32 \pm$ $2.06 \mathrm{~cm}$ ) were used in the analysis. Thirty of these participants self-identified as being right-handed, and 2 self-identified as being left-handed. The 5 remaining participants were excluded from the analysis due to using the two-hand grasping mode for an entire object presentation sequence, which did not allow for comparisons of pi numbers or of GT model parameters. ${ }^{5}$ All procedures were approved by the University's Institutional Review Board.

Materials The materials from Lopresti-Goodman et al.'s (2009) Experiment 2 were used as the experimental objects. They consisted of two sets of 41 narrow wooden planks, $2 \mathrm{~cm}$ high and $6.5 \mathrm{~cm}$ wide, ranging in length from 4.5 to $24.5 \mathrm{~cm}$ in $0.5-\mathrm{cm}$ increments, and in weight from 22 to $135 \mathrm{~g}$. These objects were painted black with their ends red and were kept behind a black curtain, and thus occluded from the participants' view, until they were presented via a small wooden paddle on a 100-cm-high table. The paddle was used to lay the objects on the presentation table in front of the participants such that they could not compare the object's widths to the experimenter's hand size, nor could they see how the experimenter would grasp each object (e.g., with one or two hands), which might have influenced the participants' actions.

Procedure Individual participants were asked to grasp and move each object from one table to another positioned $2.5 \mathrm{~m}$ away, and were told to do so at a self-selected comfortable pace $(\sim 7 \mathrm{~s} /$ plank). Participants were told that they could grasp each object with either one or two hands. Although all objects could physically be grasped with one hand by lifting the object across the $6.5-\mathrm{cm}$ width, the participants were instructed that they could only grasp the objects by the red ends, which restricted them to grasping the objects lengthwise. To ensure that participants understood how the stimuli could be grasped, the experimenter demonstrated grasping the smallest object in the set $(4.5 \mathrm{~cm})$ with one hand and grasping the largest object in the set $(24.5 \mathrm{~cm})$ with two hands.

Each participant completed two critical trials in which the planks were presented in sequence one by one in ascending and descending orders, with a random-order trial presented between them. ${ }^{6}$ The presentation sequences (ascending or descending first) were counterbalanced across participants. Each participant was randomly assigned to a

\footnotetext{
${ }^{5}$ These 5 individuals participated in the most difficult counting condition (counting back by 7). Of these 5 participants, 4 used the two-hand mode for the entire descending sequence, while 1 used two hands for the entire ascending sequence. Using this mode for the entire sequence did not allow for a calculation of $\alpha_{\mathrm{c}}$, since there was no transition between one- and two-hand grasping, and $\alpha_{\mathfrak{c}}$ values are necessary for the calculation of the GT model parameters.

${ }^{6}$ Random presentation sequences are commonly used to eliminate short-term sequential ordering effects (Tuller et al., 1994).
} 
different level of the concurrent counting task: no counting or counting back by 1,3 , or 7 starting from a number between 900 and 999. For the counting-back-by-3 and -7 conditions, the participants began backward counting from a number chosen quasirandomly, such that the last two digits did not form a number divisible by 3 or 7 , respectively. When all trials were completed, data about the participants' hand span and the maximum size of an object that could be grasped with one hand were collected.

Design and analysis The experiment had a 3 (sequence: ascending, random, and descending) $\times 4$ (counting condition: no counting or counting back by 1,3 , or 7 ) mixed design, with sequence manipulated within subjects and counting condition manipulated between subjects. The transition from one mode of grasping to another within each sequence for each participant was calculated as the mean plank length at which a participant transitioned from one- to two-hand grasping (or vice versa), divided by the participant's hand span. For example, the transition from one- to two-hand grasping in the ascending trials was calculated by taking the length of the largest plank grasped with one hand, before which all planks were grasped with one hand, and adding that number to the length of the smallest plank grasped with two hands, beyond which all planks were grasped with two hands, and dividing by 2 . This mean plank length of transition in centimeters was then divided by the participant's hand span in centimeters, resulting in a dimensionless pi number, $\alpha_{\mathrm{c}, 2}$ (LoprestiGoodman et al., 2009; Richardson et al., 2007; van der Kamp et al., 1998). This process was the same for the descending trials, resulting in a calculation of $\alpha_{\mathrm{c}, 1}$.

Predictions This experiment constituting Phase 2 was explicitly aimed at testing the predictions made by the GT model with regard to the effect of different levels of cognitive task difficulty on the GT model's parameters. Based on the parameter derivations above from LoprestiGoodman et al.'s (2009) data, it was expected that increases in counting difficulty would result in (a) increases in the amount of positive hysteresis $(\Delta \alpha)$; (b) increases in the interaction between the two grasping modes, $g$, equivalently increasing the attractor strength of both grasping modes; and (c) increases in the strength of the two-hand grasping mode's attractor, $L_{2,0}$.

Results

$\alpha_{c}$ analysis and positive hysteresis A 3 (sequence) $\times 4$ (counting condition) mixed-design ANOVA on the mean $\alpha_{\mathrm{c}}$ values revealed a significant effect of sequence, $F(2,56)=$ 36.37, $p<.001, \eta^{2}=.57$. In the ascending sequences, participants switched from one- to two-hand grasping at larger $\alpha_{\mathrm{c}}$ values $(M=0.72 \pm 0.06)$ than in either the random $(M=0.58 \pm 0.13)$ or the descending $(M=0.55 \pm 0.13)$ sequences; overall, positive hysteresis was exhibited (see Table 1 for the individual behavioral transitions). The interaction between sequence and counting condition was also significant, $F(6,56)=5.97, p<.001, \eta^{2}=.39$, with participants in the no-counting condition exhibiting mild positive hysteresis $(\Delta \alpha=.04)$, those in the 1- and 3counting conditions exhibiting moderate positive hysteresis ( $\Delta \alpha=.10$ and .23 , respectively), and those in the 7 counting condition exhibiting exaggerated positive hysteresis $(\Delta \alpha=.31)$ (see Fig. 6). These results indicate that positive hysteresis increased with the difficulty of the concurrent counting task.

Planned-comparison paired-sample $t$ tests with seven degrees of freedom for each counting condition yielded significant differences between $\alpha_{\mathrm{c}, 2}$ and $\alpha_{\mathrm{c}, 1}$ values for the 1 -, 3-, and 7-counting conditions (all $p \mathrm{~s}<.05$ ), confirming that positive hysteresis occurred. The differences in $\alpha_{\mathrm{c}}$ values for the ascending and descending sequences for the no-counting condition were not significantly different, however, $t(7)=1.75, p=.14$, indicating that despite the qualitative appearance of positive hysteresis $(\Delta \alpha=.04)$, quantitatively participants exhibited a critical-point transition. Post-hoc tests (Tukey, $p<.05$ ) revealed that within the ascending sequences, only $\alpha_{\mathrm{c}, 2}$ values for the 1- and 7counting conditions were significantly different from each other. This finding that most $\alpha_{\mathrm{c}, 2}$ values were not significantly different from one another supports the predictions made by the GT model about transitions from one- to two-hand grasping occurring at participants' biomechanical limitations. For the random sequences, only $\alpha_{\mathrm{c}}$ values for the no-counting and 7-counting conditions were significantly different from one another, and for the descending sequences, the $\alpha_{\mathrm{c}, 1}$ values for the nocounting condition was significantly different from the $\alpha_{\mathrm{c}, 1}$ values of both the 3- and 7-counting conditions. This finding supports the hypothesis that differences in the amounts of positive hysteresis for different levels of task difficulty are the result of participants using the two-hand grasping mode

Table 1 Distribution of behavior modes for individuals in the present experiment

Cognitive Task (Number Counted Back By)

\begin{tabular}{lllll}
\cline { 2 - 5 } Behavior & No Counting & 1 & 3 & 7 \\
\hline Positive hysteresis & 7 & 6 & 7 & 8 \\
Negative hysteresis & - & 1 & 1 & - \\
Critical point & 1 & 1 & - & - \\
\hline
\end{tabular}




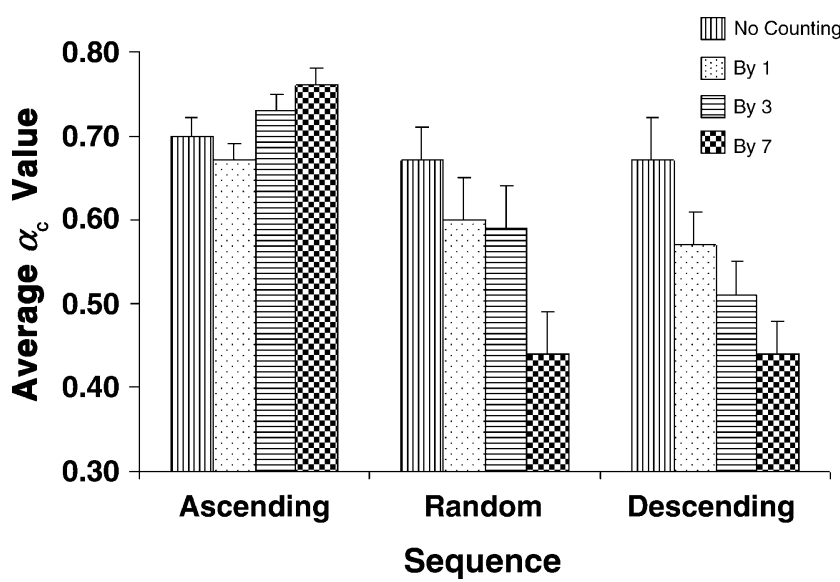

Fig. 6 The interaction of sequence and counting condition for the $\alpha_{\mathrm{c}}$ values for the Phase 2 experiment. The bars (with standard errors) represent the average transition points from one mode of grasping to another as a mean $\alpha_{\mathrm{c}}$ value for each counting condition

more often in the descending sequences (e.g., smaller $\alpha_{\mathrm{c}, 1}$ values for most difficult counting condition).

Due to the differences in the amounts of positive hysteresis exhibited for the different counting conditions, there was also a significant main effect of counting condition, $F(3,28)=3.56, p=.03, \eta^{2}=.28$. The mean $\alpha_{\mathrm{c}}$ value for the no-counting condition was larger $(M=0.68 \pm$ 0.08 ) than the mean $\alpha_{\mathrm{c}}$ value for the 1-, 3-, or 7-counting conditions $(M \mathrm{~s}=0.61 \pm 0.03,0.61 \pm 0.04$, and $0.55 \pm$ 0.04 , respectively).

GT model parameter analysis With regard to the GT model parameters, it was expected that $g$ would be significantly larger for the more difficult counting conditions than for the less difficult ones, indicating that the strength of the interaction between the two grasping modes would increase with more difficult concurrently performed counting tasks. A univariate ANOVA supported this hypothesis and revealed a significant effect of counting condition, $F(3,28)=12.18, p<.001, \eta^{2}=.57$. As expected, the $g$ values were much larger for the 7-, 3-, and 1-counting conditions $\left(M_{7}=2.38 \pm 0.63, M_{3}=1.88 \pm\right.$ $\left.0.55, M_{1}=1.33 \pm 0.29\right)$ than for the no-counting condition $(M=1.13 \pm 0.23)$ (see Fig. 7).

With regard to the $L_{2,0}$ parameter, it was expected that it would be quantitatively larger for the more difficult counting conditions. Given the marginally significant effect of counting condition in the parameter derivation of $L_{2,0}$ for Experiment 2 of Lopresti-Goodman et al. (2009), it was not certain whether or not significant differences would be found. A univariate ANOVA on the $L_{2,0}$ values for the counting conditions yielded a marginally significant effect, $F(3,28)=2.63, p=.07, \eta^{2}=$ .22 , with $L_{2,0}$ being larger for the counting conditions $\left(M_{7}=\right.$ $-0.20 \pm 0.10, M_{3}=-0.24 \pm 0.18, M_{1}=-0.23 \pm 0.13$ ) than for the no-counting condition $(M=-0.37 \pm 0.12)$ (see Fig. 8).

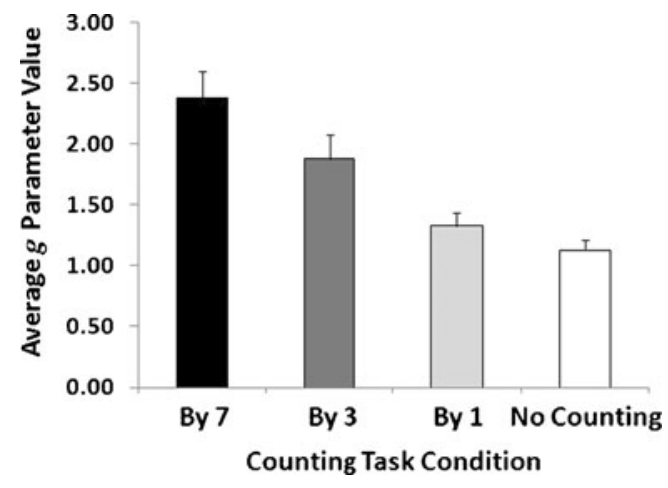

Fig. 7 The bars (with standard errors) represent the average $g$ parameter values derived for the 7 (black), 3 (dark gray), and 1 (light gray) counting conditions, as well as the no-counting condition (white), from the Phase 2 experimental data

\section{Discussion}

The aim of the experiment in Phase 2 was to determine whether the GT model was capable of accounting for differences in dynamics with systematic manipulations of a concurrently performed counting task in a grasping affordance experiment. It was expected that more difficult counting tasks would lead to exaggerated positive hysteresis (e.g., increased values of $\Delta \alpha$ ), which would result in larger values of the $g$ and $L_{2,0}$ parameters.

The results from the analysis of the $\alpha_{\mathrm{c}}$ values indicated that, in general, all participants exhibited positive hysteresis, with the degree of positive hysteresis dependent on the level of difficulty of the counting task. Specifically, positive hysteresis was exaggerated with increases in counting difficulty. Planned-comparison tests revealed that a numerical suggestion of positive hysteresis in the no-counting condition (see Fig. 6) was not significant. Instead, no-

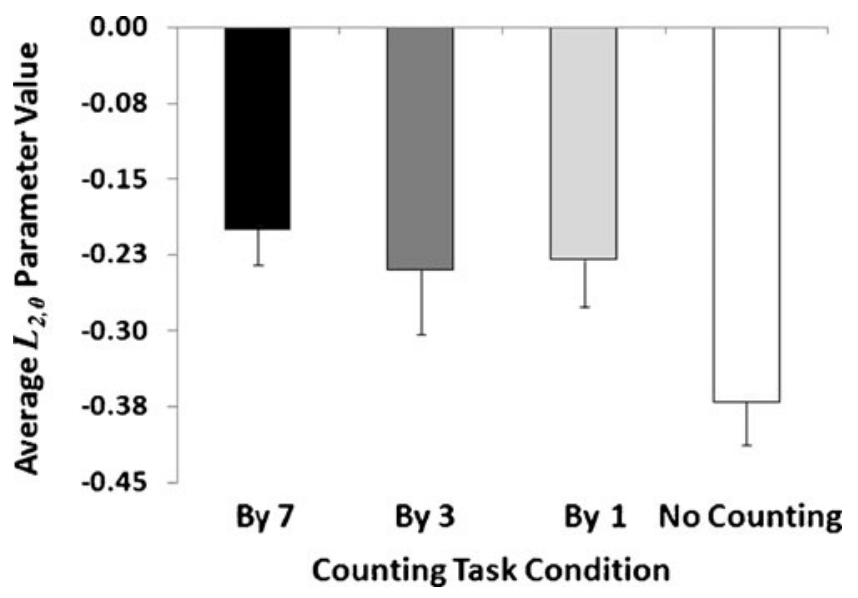

Fig. 8 The bars (with standard errors) represent the average $L_{2,0}$ parameter values derived for the 7 (black), 3 (dark gray), and 1 (light gray) counting conditions, as well as the no-counting condition (white), from the Phase 2 experimental data 
counting participants exhibited a critical-point transition. With no concurrent counting task to perform, participants could grasp and move the objects at a self-selected pace; in essence, there was no manipulation of task difficulty.

Reexamination of Lopresti-Goodman et al.'s (2009) Experiment 2 suggests a similar critical-point transition for the comparable "no-cognitive-load slow-paced" condition ( $\Delta \alpha$ values of .05 and .04 , respectively). To verify, we conducted a paired-samples $t$ test comparing the ascending $\left(\alpha_{\mathrm{c}, 2}=.71\right)$ and descending $\left(\alpha_{\mathrm{c}, 1}=.66\right) \mathrm{pi}$ numbers from Lopresti-Goodman et al.'s Experiment 2. This analysis revealed no significant difference between the two $\alpha_{\mathrm{c}}$ values, $t(11)=1.21, p>.05$. In sum, all findings with regard to increasing levels of cognitive task difficulty resulting in increasing levels of positive hysteresis were in agreement with the GT model's predictions.

Post-hoc tests on the mean $\alpha_{\mathrm{c}}$ values revealed that in the ascending sequences, the $\alpha_{\mathfrak{c}, 2}$ values for the 1- and 7counting conditions were the only ones that differed significantly. This finding supports the GT model's predictions about transitions from one- to two-hand grasping occurring near participants' biomechanical limits. ${ }^{7}$ The very small deviation in $\alpha_{\mathrm{c}, 2}$ values for this series $(S D=0.06)$ suggests that while participants switch from one- to twohand grasping at an $\alpha_{\mathrm{c}, 2}$ value that is less than 1 , this transition point is invariant across participants. Furthermore, the average $\alpha_{\mathrm{c}, 2}$ values for the ascending series in the present experiment were similar to the average $\alpha_{\mathrm{c}, 2}$ values found in Experiment 2 of Lopresti-Goodman et al. (2009; 0.72 and 0.73 , respectively), demonstrating the invariance of the oneto two-hand affordance boundary more generally.

For the descending sequences, post-hoc tests revealed that the no-counting condition's $\alpha_{\mathrm{c}, 1}$ value significantly differed from both the 3- and 7-counting conditions' $\alpha_{\mathrm{c}, 1}$ values. This finding supports the prediction made from the GT model that differences in the amount of positive hysteresis for different levels of task difficulty are mainly the result of participants using the two-hand grasping mode more often in the descending sequences. A qualitative comparison of the average $\alpha_{\mathrm{c}, 1}$ values for the descending sequences of the present experiment with the average $\alpha_{\mathrm{c}, 1}$ values for the descending sequences of Lopresti-Goodman et al.'s (2009) Experiment $2(M \mathrm{~s}=0.55 \pm 0.10$ and $0.59 \pm$ 0.15 , respectively) suggests that a constraint exists in the descending sequences, inducing a switch from two- to onehand grasping at an invariant $\alpha_{\mathrm{c}, 1}$ value.

\footnotetext{
${ }^{7}$ The biomechanical limitation must result in an $\alpha_{c}$ value less than 1 , since successfully grasping an object requires that an individual wrap his or her fingers around the ends of the object. This action limits the maximum length of an object that can be grasped with one hand to approximately $4 \mathrm{~cm}$ in length less than the hand span (cf. Richardson et al., 2007).
}

This constraint obviously does not reflect a straightforward biological limit, since two-hand grasping is afforded for all object sizes. Despite the availability of two-hand grasps, the scaling of grip configurations to an object's physical dimensions is highly systematic in both infants and adults (Cesari \& Newell, 1999; Newell, McDonald, \& Baillargeon, 1993), with the transition from one hand to two hands occurring at a common pi number. It is evident that a full understanding of the allometry of object-grip relations requires more than geometry (object size). Other factors of a possible bioenergetic nature might be involved. For example, as Choi and Mark (2004, p. 802) note, there may be a tendency to minimize overall body movement. For the present style of inquiry, theory and experiment focusing on the $\lambda_{1}>>\lambda_{2}$ region (see Fig. 2) would be needed to discern the nature of the constraint that disposes participants in the descending condition to switch from two hands to one hand at a specific $\alpha_{\mathrm{c}, 1}$ value, but this is beyond the scope of the present investigation.

Consistent with the parameter estimations for the Lopresti-Goodman et al. (2009) experiments, the strength of the two-hand grasping mode, $L_{2,0}$, marginally increased with increasing levels of task difficulty. As expected, its values were larger for the 1-, 3-, and 7-counting conditions than for the no-counting condition. The exclusion of 5 participants' data for using only the two-hand grasping mode for an entire sequence in the most difficult counting task condition lends further support for the hypothesis that the $L_{2,0}$ parameter's strength increases with increasing cognitive task difficulty. These participants found the task so difficult that they used the two-hand mode for the entire sequence.

Given the reliable impact of task difficulty on the $g$ parameter and the weaker impact on $L_{2,0}$, it may be inferred that the strength of the interaction of the two grasping modes dictates the behavioral dynamics of transitions in grasping. An evaluation of this claim can be made by deriving an explicit equation that relates $\Delta \alpha_{\mathrm{c}}$ to $g$ and $L_{2,0}$. A detailed calculation shows that

$\Delta \alpha_{\mathrm{c}}=[(g-1) /(g+1)]\left(1+L_{2,0}\right)$

or, equivalently, $\Delta \alpha=z^{*} w$, where $z$ is a function that only depends on $g$, and $w$ is a function that only depends on $L_{2,0}$. For example, a comparison of the $\Delta \alpha$ values for the nocounting and the counting-back-by-7 conditions indicates that the amount of positive hysteresis for the counting-back-by-7 condition is 5.9 times greater than that for the no-counting condition (see Appx. D). By determining how much of this difference is attributable to the $z$ parameter (and consequently determining the effect of $g$ ) and how much is attributable to the $w$ parameter (the effect of $L_{2,0}$ ), it becomes evident that the $z$ component or, equivalently, the $g$ parameter accounts 
for almost all of the difference in the amount of positive hysteresis exhibited in these two conditions $(\sim 80 \%)$.

\section{Conclusions: Dual tasking, parameter modulation, and the organism-environment system}

The primary goal of the present study was to advance the experimental investigation of affordances by incorporating the investigation within a dynamical systems framework. Whereas the results of previous affordance experiments have been interpreted as instances of self-organized dynamics (e.g., Fitzpatrick et al., 1994; Hirose \& Nishio, 2001; Lopresti-Goodman et al., 2009; Richardson et al., 2007; van der Kamp et al., 1998), the present study is the first to express and evaluate this interpretation formally by means of a dynamical model. The GT model of the affordance "graspable" proposed by Frank et al. (2009) is an extension of the thesis that a deep analogy exists between pattern perception and the physical principles of pattern formation (Haken, 2004).

Our analyses of the GT model parameters suggested that performance of an unrelated counting task in parallel with the grasping task modulated the attractors governing the grasp-transition dynamics in two ways: It shifted their locations and changed their interaction strength and stability. Specifically, our results revealed that increased counting difficulty (increased information reduction, in the Shannon sense) was accompanied primarily by an increase in the strength of the competitive interaction between the one- and two-hand grasping modes (i.e., larger values of $g$ ) and by an increase in the stability of the two-hand grasping mode's attractor (i.e., larger values of $L_{2,0}$ ).

Analogous effects in coordination dynamics

The dynamics of bimanual rhythmic coordination have similarly been developed from the self-organizing principles of pattern formation (Haken, Kelso, \& Bunz, 1985; Kelso, 1995). The present finding, that the concurrent performance of an unrelated counting task reparameterizes grasping dynamics, complements the results of experiments suggesting that the dynamics of bimanual rhythmic coordination are reparameterized by parallel cognitive activity. In these rhythmic coordination experiments, however, the equivalents of $\xi, \alpha$, and $\lambda$ (the order, control, and attention or availability parameters, respectively) are fixed within a trial, and a particular cognitive activity at a prescribed level is performed in parallel with the coordination task within each trial. Across trials, one or more of the order, control, or availability parameters, depending on the experiment, are manipulated. In sum, these experiments are not designed to induce transitions.
The focus of these experiments has been cognitive activity's influence on the attractor or stable state of 1:1 frequency locking of two limbs. The attractor is characterized by its location on the relative phase continuum, its strength, and its noise. Parallel performance of the Posner and Rossman (1965) tasks has been shown to shift coordination attractors in proportion to the degree of information reduction (Pellecchia \& Turvey, 2001). A more detailed analysis shows that parallel performance of counting backward (by 3 s) shifts an attractor to a new value and amplifies its noise (i.e., the stable state fluctuates more) but preserves its strength (i.e., the tendency for the coordination to settle back onto the stable state following a perturbation) (Pellecchia, Shockley, \& Turvey, 2005). Similar outcomes hold when the parallel activity is memory related (Shockley \& Turvey, 2005). With respect to encoding a list of unrelated words, encoding affects parallel rhythmic coordination (new attractor, more noise), and parallel rhythmic coordination affects encoding. With respect to retrieving a list of previously encoded unrelated words, retrieval affects parallel rhythmic coordination (new attractor, more noise), but not vice versa.

Of particular relevance is the finding that when bimanual rhythmic coordination is performed in parallel with the semantic memory task of retrieving members of a category, retrieval is impaired and the dynamics of coordination are altered (Shockley \& Turvey, 2006). Excluded from Shockley and Turvey's experiment, however, were commonly identified sources of specific intertask interference (Bourke, 1997; Bourke, Duncan, \& Nimmo-Smith, 1996), such as shared input modality (Treisman \& Davies, 1973) or similar semantic domain (Hirst \& Kalmar, 1987). A factor common to semantic retrieval and rhythmic coordination is implied by their results, but the conventional appeal to limited cognitive resources ${ }^{8}$ fails to address the intricate pattern of mutual influences between the two activities. It has been suggested that stability is a more promising candidate for the implied general factor influenced by parallel performance of both tasks (Shockley \& Turvey, 2006). The seeming mix of monitoring and control processes constituting semantic retrieval (Baddeley, 1998; Koriat, 2000) suggests that semantic retrieval, like coordination, could be framed in dynamical terms. Semantic retrieval has a characteristic dynamic, as noted originally by Bousfield and Sedgewick (1944) and elaborated recently by Rhodes and colleagues (Frank \& Rhodes, 2010; Rhodes \& Turvey,

\footnotetext{
${ }^{8}$ Questions about dual-task limitations tend to dominate current theorizing (Hazeltine, Ruthruff, \& Remington, 2006). Is the parallel performance of two distinct tasks subject to limitations in central processing? Do central limitations lead to serial processing of the two tasks? Does central interference depend only on the duration of competition for central capacity, or does it also depend critically on the specific content of the two tasks?
} 
2007). The dual-task performance of semantic retrieval and coordination could, therefore, be interpreted as a coupling of the two dynamics, a coupling that might be expected to entail parameter changes in retrieval dynamics paralleling the identified parameter changes in coordination dynamics. On the hypothesis advanced above, the criterion for these dual changes would be enhanced stability of the coupled system.

The latter ideas are in keeping with Neumann's (1987) suggestion that dual-task performance is a higher-order skill with its own parameterization, and perhaps with Logan and Gordon's (2001) notion that a dual task composed of cognitive and behavioral activities entails a higher-level control that ensures the successful fit of the subordinate activities by reconfiguring one or both of them through the manipulation of their parameters.

How do these conceptions apply to the dual tasking in grasping dynamics (i.e., to the data on grasping transitions obtained in Phase 2)? If information reduction tasks were dynamic systems, bringing new functional terms to the GT model, then it would have to be assumed that these new terms modulated the parameters of the grasping dynamics commensurate with the magnitude of information reduction. The challenge posed for theory and modeling would be how to extend the principles underwriting the formulation of the GT model (summarized in Haken, 2004) to the domain of serial and nested mental operations of the kind represented minimally by the counting tasks of the Phase 2 experiment.

The organism-environment system and future inquiry

The GT dynamical system incorporates the complementary relation that exists between the relevant properties of the tobe-grasped objects (i.e., object length or size) and the relevant properties of the individual's action system (i.e., hand size). It is this person-environment relation (more precisely, the information that specifies this person-environment relation) that constrains and guides the grasping behavior of an individual. The attractors and repellers studied in the present research do not characterize states of body or brain, but rather states of the organism-environment system.

The present GT modeling, methodology, and focus on affordances are in concert, therefore, with the thesis that perceiving, acting, and knowing emerge from the interplay of body, brain, and environmental surroundings (e.g., Calvo \& Gomila, 2008; Clancey, 1997; Clark, 1997, 1999) and are approachable through the tools of dynamical-systems theory (e.g., Beer, 1995a, 1995b, 2009; Calvo \& Gomila, 2008). For Järvilehto (1998, 2009), adopting the perspective of organism $(\mathrm{O})$ and environment $(\mathrm{E})$ as one system carries three implications: (1) Explanations of behavior have to be in terms of system reorganization (as opposed to a movement of $\mathrm{O}$ or an interaction of $\mathrm{O}$ and $\mathrm{E}$ ). (2) Activities typically termed "mental" have to be understood as aspectual differences in system organization (as opposed to local processes of $\mathrm{O}$ ). (3) The focus of analysis is neither behavior nor mental activity, as such, but rather the $\mathrm{O}-\mathrm{E}$ system's results or outcomes.

Our results approximate an understanding of implications (1) and (3) above; future studies may be directed at evaluating implication (2) within the GT model and its focus upon E defined through affordances. Most immediately, they should be aimed at (a) determining whether or not the GT model is able to account for the negative hysteresis of grasping transitions when the classification "graspable" is verbal and not a selective action of the upper limbs, and (b) whether the model can be generalized to account for positive and negative hysteresis exhibited in affordance tasks other than grasping (e.g., "sit-on-able," "step-over-able," "reach-with-able," and so on).

Author Note This work was conducted as part of the doctoral dissertation of S.M.L.-G. while at the Center for the Ecological Study of Perception and Action at the University of Connecticut. The authors acknowledge Claudia Carello, Jeffrey Kinsella-Shaw, and Michael J. Richardson, who served as co-advisors on S.M.L.-G.'s dissertation committee. The authors also thank Elliot Saltzman and Leonard Mark for feedback on the dissertation proposal paper. The experimental data collected by Lopresti-Goodman et al. (2009), used for the parameter derivations in the present study, were supported by National Science Foundation Grant BSC-0342802, awarded to Kerry L. Marsh, Claudia Carello, Reuben M. Baron, and Michael J. Richardson.

\section{Appendix A}

By substituting Eqs. (6) and (7) into Eqs. (1) and (2), we obtain

$\dot{\xi}_{1}=\beta(1-\alpha) \xi_{1}-B \xi_{2}^{2} \xi_{1}-C \xi_{1}\left(\xi_{1}^{2}+\xi_{2}^{2}\right)$

and

$\dot{\xi}_{2}=\left(\lambda_{2,0}+\alpha \beta\right) \xi_{2}-B \xi_{1}^{2} \xi_{2}-C \xi_{2}\left(\xi_{1}^{2}+\xi_{2}^{2}\right)$.

We divide these equations by $\beta$ in order to obtain

$\frac{1}{\beta} \dot{\xi}_{1}=(1-\alpha) \xi_{1}-\frac{B}{\beta} \xi_{2}^{2} \xi_{1}-\frac{C}{\beta} \xi_{1}\left(\xi_{1}^{2}+\xi_{2}^{2}\right)$,

and

$\frac{1}{\beta} \dot{\xi}_{2}=\left(L_{2,0}+\alpha\right) \xi_{2}-\frac{B}{\beta} \xi_{1}^{2} \xi_{2}-\frac{C}{\beta} \xi_{2}\left(\xi_{1}^{2}+\xi_{2}^{2}\right)$.

We rescale time $(t)$ by the parameter $\beta$; that is, we introduce a new time variable $t^{\prime}=t \beta$, which implies that

$\frac{1}{\beta} \frac{d}{d t} \xi_{1}=\frac{d}{d t^{\prime}} \xi_{1}, \frac{1}{\beta} \frac{d}{d t} \xi_{2}=\frac{d}{d t^{\prime}} \xi_{2}$. 
Consequently, Eqs. (16) and (17) become

$\dot{\xi}_{1}=(1-\alpha) \xi_{1}-\frac{B}{\beta} \xi_{2}^{2} \xi_{1}-\frac{C}{\beta} \xi_{1}\left(\xi_{1}^{2}+\xi_{2}^{2}\right)$

and

$\dot{\xi}_{2}=\left(L_{2,0}+\alpha\right) \xi_{2}-\frac{B}{\beta} \xi_{1}^{2} \xi_{2}-\frac{C}{\beta} \xi_{2}\left(\xi_{1}^{2}+\xi_{2}^{2}\right)$,

where the over-dot now refers to differentiation with respect to $t^{\prime}$ rather than with respect to $t$. Next, we note that the following relation holds:

$\frac{B}{\beta} \xi_{2}^{2} \xi_{1}+\frac{C}{\beta} \xi_{2}^{2} \xi_{1}=\frac{B+C}{\beta} \xi_{2}^{2} \xi_{1}=\frac{C}{\beta}\left(1+\frac{B}{C}\right) \xi_{2}^{2} \xi_{1}=\frac{C}{\beta} g \xi_{2}^{2} \xi_{1}$,

and

$\frac{B}{\beta} \xi_{1}^{2} \xi_{2}+\frac{C}{\beta} \xi_{1}^{2} \xi_{2}=\frac{B+C}{\beta} \xi_{1}^{2} \xi_{2}=\frac{C}{\beta}\left(1+\frac{B}{C}\right) \xi_{1}^{2} \xi_{2}=\frac{C}{\beta} g \xi_{1}^{2} \xi_{2}$.

Consequently, Eqs. (19) and (20) can equivalently be expressed as

$\dot{\xi}_{1}=(1-\alpha) \xi_{1}-\frac{C}{\beta} g \xi_{2}^{2} \xi_{1}-\frac{C}{\beta} \xi_{1}^{3}$,

and

$\dot{\xi}_{2}=\left(L_{2,0}+\alpha\right) \xi_{2}-\frac{C}{\beta} g \xi_{1}^{2} \xi_{2}-\frac{C}{\beta} \xi_{2}^{3}$.

In order to show how to rescale the amplitudes, we cast Eqs. (23) and (24) into the form

$\dot{\xi}_{1}=\xi_{1}\left\{(1-\alpha)-g \frac{C}{\beta} \xi_{2}^{2}-\frac{C}{\beta} \xi_{1}^{2}\right\}$,

and

$\dot{\xi}_{2}=\xi_{2}\left\{\left(L_{2,0}+\alpha\right)-g \frac{C}{\beta} \xi_{1}^{2}-\frac{C}{\beta} \xi_{2}^{2}\right\}$.

As mentioned in the main text, we rescale the amplitudes $\xi_{1}$ and $\xi_{2}$ with the square root of $C / \beta$. That is, we introduce the new variables $\xi_{1}^{\prime}$ and $\xi_{2}^{\prime}$ defined by

$\xi_{1}^{\prime}=\sqrt{\frac{C}{\beta} \xi_{1}} \quad, \quad \xi_{2}^{\prime}=\sqrt{\frac{C}{\beta} \xi_{2}}$.

Then, Eqs. (25) and (26) read

$\dot{\xi}_{1}^{\prime}=\xi_{1}^{\prime}\left\{(1-\alpha)-g\left(\xi_{2}^{\prime}\right)^{2}-\left(\xi_{1}^{\prime}\right)^{2}\right\}$,

and

$\dot{\xi}_{2}^{\prime}=\xi_{2}^{\prime}\left\{\left(L_{2,0}+\alpha\right)-g\left(\xi_{1}^{\prime}\right)^{2}-\left(\xi_{2}^{\prime}\right)^{2}\right\}$.
The rescaled model equations involve only two parameters, $L_{2,0}$ and $g$. For the sake of readability, we next drop the primes. Subsequently, we write out the terms in Eqs. (28) and (29), and in doing so obtain Eqs. (11) and (12) mentioned in the main text.

\section{Appendix B}

We can estimate $g$ and $L_{2,0}$ on the basis of LoprestiGoodman et al.'s (2009) experimental observations of $\alpha_{\mathrm{c}, 1}$ and $\alpha_{\mathrm{c}, 2}$. To this end, we transform Eq. (13) of Frank et al. (2009). Equation (13) involves two relations that read

$\alpha_{c, 1}=\frac{1}{\beta(1+g)}\left(\lambda_{1,0}-g \lambda_{2,0}\right)$,

and

$\alpha_{c, 2}=\frac{1}{\beta(1+g)}\left(g \lambda_{1,0}-\lambda_{2,0}\right)$,

As explained in detail in Frank et al. (2009), a natural choice for the parameter $\lambda_{1,0}$ occurring in Eqs. (30) and (31) is $\lambda_{1,0}=\beta$. In particular, in this case the linear relationship $\lambda_{1}=\lambda_{1,0}-\beta \alpha$ becomes Eq. (6) mentioned in the introduction. Substituting $L_{2,0}=\lambda_{2,0} / \beta$ (see the introduction) and $\lambda_{1,0} / \beta=1$ into Eqs. (30) and (31), respectively, we obtain

$\alpha_{c, 1}=\frac{1-g L_{2,0}}{1+g}$

and

$\alpha_{c, 2}=\frac{g-L_{2,0}}{1+g}$.

We add Eqs. (32) and (33), which gives us

$\alpha_{c, 1}+\alpha_{c, 2}=\frac{1-g L_{2,0}+g-L_{2,0}}{1+g}=\frac{1+g-(1+g) L_{2,0}}{1+g}=1-L_{2,0}$.

Consequently, we can compute $L_{2,0}$ from $\alpha_{\mathrm{c}, 1}$ and $\alpha_{\mathrm{c}, 2}$ :

$L_{2,0}=1-\alpha_{c, 1}-\alpha_{c, 2}$.

Next, we substitute Eq. (35) into Eq. (33):

$\alpha_{c, 2}=\frac{g-L_{2,0}}{1+g}=\frac{g-1+\alpha_{c, 1}+\alpha_{c, 2}}{1+g} \Rightarrow \alpha_{c, 2}(1+g)=g+\alpha_{c, 1}+\alpha_{c, 2}-1$.

We collect all terms involving the parameter $g$ on the left side of the equal sign, and thus obtain

$\alpha_{c, 2}+g \alpha_{c, 2}-g=\alpha_{c, 1}+\alpha_{c, 2}-1 \Rightarrow g\left(\alpha_{c, 2}-1\right)=\alpha_{c, 1}-1$ 
which leads to our final result

$g=\frac{\alpha_{c, 1}-1}{\alpha_{c, 2}-1}=\frac{1-\alpha_{c, 1}}{1-\alpha_{c, 2}}$.

In our study, we use Eqs. (35) and (38) to estimate the model parameters $g$ and $L_{2,0}$ from the observed $\alpha_{\mathrm{c}}$ values.

\section{Appendix C}

According to Posner and Rossman (1965), the amount of information reduced is directly related to task difficulty and can be calculated by considering the reduction of the size of the population of numbers with which a participant deals. When counting back by $1 \mathrm{~s}$ starting from 100, the initial population of numbers a participant deals with is 0 through 99. Counting back by $1 \mathrm{~s}$ therefore preserves all of the information in the sequence of 100 numbers, which means a reduction of 0 bits of information. If numbers are skippedas in counting backward by any number greater than $1-$ there is selection of a part of the information in the series, meaning that there is a reduction in the series's information.

In counting back by $1 \mathrm{~s}$, the average population of numbers decreases over the course of the task. It approximates $50(99 / 2=$ 49.5). In order to calculate the bits of information in each subsequent step during this maximum information-preserving task, we take $\log _{2}$ of 50 , which equals 5.64 bits. Posner and Rossman (1965) concluded that counting backward by $3 \mathrm{~s}$ from 100 requires the selection of one-third of the information in this series, so $5.64 / 3=1.88$ bits, which is a reduction of 3.76 bits of information per step.

Pellecchia and Turvey (2001) extended this logic to determine the bits of information reduced when counting backward by $3 \mathrm{~s}$ from a randomly chosen three-digit number. They determined that this task led to the reduction of 5.9 bits of information per step. In the present experiment, trials lasted on average $5 \mathrm{~min}$. To avoid having participants count down to a number less than 0 during this time frame, we set the range of three-digit numbers from which the participants began to 900-999. This gives an approximation of the population size of 475 (950/2); therefore, counting back by $1 \mathrm{~s}$ would result in 8.89 bits of information per step. Counting back by $7 \mathrm{~s}$ requires a selection of one-seventh of the information at each step. That is $8.89 / 7=1.27$ bits, yielding a reduction of 7.62 bits.

\section{Appendix D}

The amount of hysteresis $(\Delta \alpha)$ exhibited in an experimental condition can be calculated from Eqs. (32) and (33). To determine the contribution of each of the GT model parameters to $\Delta \alpha$, Eqs. (32) and (33) can be converted into an equation representing a $g$ component, $z$, and an $L_{2}$ component, $w$ (see Eq. 13). A comparison of the $z$ and $w$ parameters on $\Delta \alpha$ for the most difficult cognitive-task condition (counting back by $7 \mathrm{~s}$ ) and the least difficult cognitive-task condition (no counting) for the present experiment will be considered.

For the counting-back-by-7 condition, Eq. (13) yields a $z$ value of 0.390 , a $w$ value of 0.800 , and a $\Delta \alpha$ value of .312. For the no-counting condition, Eq. (13) yields a $z$ value of 0.083 , a $w$ value of 0.640 , and a $\Delta \alpha$ value of .053. A comparison of the $\Delta \alpha$ values for the two conditions $\left(M_{7}=.312\right.$ and $\left.M_{\text {NoCounting }}=.053\right)$ reveals that participants in the counting-back-by-7 condition exhibited 5.9 times more hysteresis than those in the no-counting condition. To determine which GT model parameter contributed more to the amount of hysteresis exhibited, a comparison of the $z$ and $w$ components for the two conditions reveals that $z$ for the counting-backby-7 condition was 4.7 times larger than $z$ for the nocounting condition $\left(M_{7}=0.390\right.$ and $\left.M_{\text {NoCounting }}=0.083\right)$, while the $w$ parameter was only 1.4 times larger for the 7counting than for the no-counting condition $\left(M_{7}=0.800\right.$ and $\left.M_{\text {NoCounting }}=0.640\right)$. From this comparison, it can be concluded that the $g$ parameter contributes more to the amount of hysteresis exhibited than does $L_{2}$, given that the $z$ parameter accounted for almost $80 \%$ of the differences in $\Delta \alpha[(4.7 / 5.9) \times 100=79.66 \%]$.

\section{References}

Baddeley, A. D. (1998). Human memory: Theory and practice. Boston: Allyn \& Bacon.

Beer, R. D. (1995a). Computational and dynamical languages for autonomous agents. In R. F. Port \& T. van Gelder (Eds.), Mind as motion: Explorations in the dynamics of cognition (pp. 121147). Cambridge, MA: MIT Press.

Beer, R. D. (1995b). A dynamical systems perspective on agentenvironment interaction. Artificial Intelligence, 72, 173-215. doi:10.1016/0004-3702(94)00005-L

Beer, R. D. (2009). Beyond control: The dynamics of brain-bodyenvironment interaction in motor systems. In D. Sternad (Ed.), Progress in motor control: A multidisciplinary perspective (pp. 7-24). New York: Springer.

Bestehorn, M., \& Friedrich, R. (1999). Rotationally invariant order parameter equations for natural patterns in nonequilibrium systems. Physical Review E, 59, 2642-2652.

Bestehorn, M., Friedrich, R., \& Haken, H. (1989). Two-dimensional traveling wave patterns in nonequilibrium systems. Zeitschrift für Physik B, 75, 265-274.

Bestehorn, M., \& Haken, H. (1991). Associative memory of a dynamical system: An example ofthe convection instability. Zeitschrift für Physik B, 82, 305-308.

Bourke, P. A. (1997). Measuring attentional demand in continuous dual-task performance. The Quarterly Journal of Experimental Psychology, 50A, 821-840. 
Bourke, P. A., Duncan, J., \& Nimmo-Smith, I. (1996). A general factor involved in dual task performance decrement. The Quarterly Journal of Experimental Psychology, 49A, 525-545.

Bousfield, W. A., \& Sedgewick, C. H. W. (1944). An analysis of sequences of restricted associative responses. The Journal of General Psychology, 30, 149-165.

Bressloff, P. C., \& Roper, P. (1998). Stochastic dynamics of the diffusive Haken model with subthreshold periodic forcing. Physical Review E, 58, 2282-2287.

Calvo, P., \& Gomila, A. (2008). Handbook of cognitive science: An embodied approach. Amsterdam: Elsevier Science.

Cesari, P., \& Newell, K. M. (1999). Body-scaled transitions in human grip configurations. Journal of Experimental Psychology. Human Perception and Performance, 26, 1657-1668.

Cesari, P., \& Newell, K. M. (2000). Body scaling of grip configurations in children aged 6-12 years. Developmental Psychobiology, 36, 301-310.

Chemero, A., \& Turvey, M. (2007). Gibsonian affordances for roboticists. Adaptive Behavior, 15, 473-480. doi:10.1177/1059712307085098

Choi, H. J., \& Mark, L. S. (2004). Scaling affordances for human reach actions. Human Movement Science, 23, 785-806.

Clancey, W. J. (1997). Situated cognition: On human knowledge and computer representations. New York: Cambridge University Press.

Clark, A. (1997). The dynamical challenge. Cognitive Science, 21, 461-481. doi:10.1016/S0364-0213(99)80030-5

Clark, A. (1999). Embodiment: From fish to fantasy. Trends in Cognitive Sciences, 3, 345-351.

Ditzinger, T., \& Haken, H. (1989). Oscillations in the perception of ambiguous patterns. Biological Cybernetics, 61, 279-287.

Ditzinger, T., Tuller, B., Haken, H., \& Kelso, J. A. S. (1997). A synergetic model of the verbal transformation effect. Biological Cybernetics, 77, 31-40.

Fitzpatrick, P., Carello, C., Schmidt, R. C., \& Corey, D. (1994). Haptic and visual perception of an affordance for upright posture. Ecological Psychology, 6, 265-287. doi:10.1207/s15326969eco0604_2

Frank, T. D. (2009). On a multistable competitive network model in the case of a inhomogeneous growth rate spectrum: With an application to priming. Physics Letters A, 373, 4127-4133.

Frank, T. D., \& Rhodes, T. (2010). Micro-dynamic associated with twostate nonlinear Markov processes: With an application to free recall. Fluctuation and Noise Letters, 10, 41-58. doi:10.1142/ S0219477511000387

Frank, T. D., Richardson, M. J., Lopresti-Goodman, S. M., \& Turvey, M. T. (2009). Order parameter dynamics of body-scaled hysteresis and mode transitions in grasping behavior. Journal of Biological Physics, 35, 127-147.

Frank, T. D., van der Kamp, J., \& Savelsbergh, G. J. P. (2010). On a multistable dynamic model of behavioral and perceptual infant development. Developmental Psychobiology, 52, 352-371. doi: 10.1002/dev.20431

Fuchs, A., \& Haken, H. (1988a). Pattern recognition and associative memory as dynamical processes in a synergetic system: I. Translational invariance, selective attention and decomposition of scene. Biological Cybernetics, 60, 17-22.

Fuchs, A., \& Haken, H. (1988b). Pattern recognition and associative memory as dynamical processes in a synergetic system: II. Decomposition of complex scenes, simultaneous invariance with respect to translation, rotation, and scaling. Biological Cybernetics, 60, 107-109.

Gibson, J. J. (1979). The ecological approach to visual perception. Boston: Houghton Mifflin.

Haken, H. (1988). Nonequilibrium phase transitions in pattern recognition and associative memory. Zeitschrift für Physik B, 70, 121-123.

Haken, H. (1991). Synergetic computers and cognition. Berlin: Springer.
Haken, H. (2004). Synergetics: Introduction and Advanced Topics. Berlin: Springer.

Haken, H., Kelso, J. A. S., \& Bunz, H. (1985). A theoretical model of phase transitions in human hand movements. Biological Cybernetics, 51, 347-356.

Hazeltine, E., Ruthruff, E., \& Remington, R. W. (2006). The role of input and output modality pairings in dual-task performance: Evidence for content-dependent central interference. Cognitive Psychology, 52, 291-345.

Hirose, N., \& Nishio, A. (2001). The process of adaptation to perceiving new action capabilities. Ecological Psychology, 13, 49-69. doi:10.1207/S15326969ECO1301_3

Hirst, W., \& Kalmar, D. (1987). Characterizing attentional resources. Journal of Experimental Psychology. General, 116, 68-81. doi:10.1037/0096-3445.116.1.68

Järvilehto, T. (1998). The theory of the organism-environment system: I. Description of the theory. Integrative Physiological and Behavioral Science, 33, 321-334. doi:10.1007/BF02688700

Järvilehto, T. (2009). The theory of the organism-environment system as a basis of experimental work in psychology. Ecological Psychology, 21, 112-120. doi:10.1080/10407410902877066

Kelso, J. A. S. (1995). Dynamic patterns. Cambridge, MA: MIT Press.

Koriat, A. (2000). Control processes in remembering. In E. Tulving \& F. I. M. Craik (Eds.), The Oxford handbook of memory (pp. 333346). New York: Oxford University Press.

Logan, G. D., \& Gordon, R. D. (2001). Executive control of visual attention in dual-task situations. Psychological Review, 108, 393-434.

Lopresti-Goodman, S. M., Richardson, M. J., Baron, R. M., Carello, C., \& Marsh, K. L. (2009). Task constraints on affordance boundaries. Motor Control, 13, 69-83.

Merk, I., \& Schnakenberg, J. (2002). A stochastic model of multistable visual perception. Biological Cybernetics, 86, 111-116.

Murray, J. D. (1993). Mathematical biology: An introduction. New York: Springer.

Nagao, N., Haruhiko, H., \& Matsui, N. (2000). A neural chaos model of multistable perception. Neural Processing Letters, 12, 267-276.

Neumann, O. (1987). Beyond capacity: A functional view of attention. In O. Neumann \& A. F. Sanders (Eds.), Perspectives on perception and action (pp. 361-394). Hillsdale, NJ: Erlbaum.

Newell, K. M., McDonald, P. V., \& Baillargeon, R. (1993). Bodyscale and infant grip configurations. Developmental Psychobiology, 26, 195-205.

Newell, K. M., Scully, D. M., Tenenbaum, F., \& Hardiman, S. (1989). Body-scale and the development of prehension. Developmental Psychobiology, 64, 469-475.

Pellecchia, G., Shockley, K., \& Turvey, M. T. (2005). Concurrent cognitive task modulates coordination dynamics. Cognitive Science, 29, 531-557. doi:10.1207/s15516709cog0000_12

Pellecchia, G., \& Turvey, M. T. (2001). Cognitive activity shifts the attractors of bimanual rhythmic coordination. Journal of Motor Behavior, 33, 9-15. doi:10.1080/00222890109601898

Posner, M. I. (1964). Information reduction in the analysis of a sequential task. Psychological Review, 71, 491-504. doi:10.1037/h0041120

Posner, M. I., \& Rossman, E. (1965). Effect of size and location of informational transforms on short-term retention. Journal of Experimental Psychology, 70, 496-505. doi:10.1037/h0022545

Pufall, P. B., \& Dunbar, C. (1992). Perceiving whether or not the world affords stepping onto and over: A developmental study. Ecological Psychology, 4, 17-38.

Rhodes, T., \& Turvey, M. T. (2007). Human memory retrieval as Lévy foraging. Physica A, 385, 255-260.

Richardson, M. J., Marsh, K. L., \& Baron, R. M. (2007). Judging and actualizing interpersonal and intrapersonal affordances. Journal of Experimental Psychology. Human Perception and Performance, 33, 845-859. doi:10.1037/0096-1523.33.4.845 
Richardson, M. J., Shockley, K., Fajen, B., Riley, M., \& Turvey, M. T. (2008). Ecological psychology: Six principles for an embodiedembedded approach to behavior. In P. Calvo \& A. Gomila (Eds.), Handbook of cognitive science: An embodied approach (pp. 161-197). Amsterdam: Elsevier Science.

Rubin, E. (1915). Synsoplevede figurer. Copenhagen: Gyldenhal.

Shockley, K., \& Turvey, M. T. (2005). Encoding and retrieval during bimanual rhythmic coordination. Journal of Experimental Psychology. Learning, Memory, and Cognition, 31, 980-990. doi:10.1037/0278-7393.31.5.980

Shockley, K., \& Turvey, M. T. (2006). Dual task influences on retrieval from semantic memory and coordination dynamics. Psychonomic Bulletin \& Review, 13, 985-990.

Strogatz, S. H. (1994). Nonlinear dynamics and chaos: With applications to physics, biology, chemistry, and engineering. Reading, MA: Addison-Wesley.
Treisman, A., \& Davies, A. (1973). Divided attention to ear and eye. In S. Kornblum (Ed.), Attention and performance IV (pp. 101117). New York: Academic Press.

Tuller, B., Case, P., Ding, M., \& Kelso, J. A. (1994). The nonlinear dynamics of speech categorization. Journal of Experimental Psychology. Human Perception and Performance, 20, 3-16. doi:10.1037/0096-1523.20.1.3

van der Kamp, J., Savelsbergh, G. J. P., \& Davis, W. E. (1998). Bodyscaled ratio as a control parameter for prehension in 5- to 9-year-old children. Developmental Psychobiology, 33, 351-361. doi:10.1002/ (SICI)1098-2302(199812)33:4<351::AID-DEV6>3.0.CO;2-P

Warren, W. H. (1984). Perceiving affordances: Visual guidance of stair climbing. Journal of Experimental Psychology. Human Perception and Performance, 10, 683-703. doi:10.1037/0096-1523.10.5.683

Warren, W. H. (2006). Dynamics of perception and action. Psychological Review, 113, 358-389. doi:10.1037/0033-295X.113.2.358 